\title{
Analisi farmacoeconomica del trattamento di pazienti psicotici cronici con olanzapina, antipsicotici atipici e neurolettici tipici: uno studio regionale

Felice Vadruccio*, Giovanni Biricolti§, Leonardo Mendolicchio*

\begin{abstract}
BACKGROUND: Psychotic disorders are a ravaging and costly mental illness. Treatment of these disorders involves pharmacological and non pharmacological resources. Pharmacological therapy with antipsychotic drugs contribute strongly to relieve psychotic patients symptoms. By the end of 90's the new atypical antipsychotic have been introduced. This kind of drugs is supposed to be more effective but also more expensive compared with the old typical neuroleptics.

OBJECTIVE: To compare costs and outcomes associated with 12 months treatment of psychotic disorders using typical and atypical antipsychotics in addition to psychiatric services. Moreover to extend the same comparison among the atypical drugs currently used in the clinical practice (olanzapine, risperidone, quetiapine and clozapine). METHODS AND PATIENTS: A multicentre observational study was carried out in 131 patients affected by psychotic disorders (schizophrenia and bipolar). Data were collected with reference to patients followed by several Psychiatric Services of Regione Puglia (Italy). Patients were classified in five groups (typical neuroleptics, olanzapine, risperidone, quetiapine and clozapine) according to their main antipsychotic therapy. Treatment outcomes had been assessed during 12 months of observation considering the patients improvement in their work and social functioning. For these patients we analyzed pharmacological, non pharmacological (medical/ nurse visits, social assistance, rehabilitative sessions) and hospital interventions, choosing the perspective of the Italian Mental Health Centers for costs attribution. Moreover indirect costs (caregiver) and private assistance to the patient had been evaluated for each group of treatment. The study is based on the observation of real clinical behaviours; therefore patients are not randomised to different treatments.

RESULTS: The analysis of treatment outcomes didn't generate significant differences among the groups despite a positive trend in terms of work functioning in favor of olanzapine. The pharmacological interventionts evidentiated an economic significant advantage $(\mathrm{p}<0,05)$ in the typical group, compared to all atypical groups, due to the cheaper cost of these drugs. However the overall costs of treatment (pharmacological and non pharmacological resources) didn't evidentiate significant differences between typical and atypical agents. The comparison among atypical drugs showed a significant $(\mathrm{p}=0,0426)$ overall cost advantage of olanzapine group (8.843 euros) compared to quetiapine group (12.344 euros). Moreover the finding from this study suggest that olanzapine leads to reduce in the long term the caregiver costs avoiding at the same time the use of the private assistance for the chronic psychotic patient.

CONCLUSIONS: Within the local clinical context, olanzapine appears to provide advantages over the other antipsychotics considering the reduction, statistically significant, in total healthcare resources utilization and savings in terms of costs of caregiver and private assistance related to its use.
\end{abstract}

Keywords: psychotic disorders, costs, outcomes, typical neuroleptics, atypical antipsychotics Farmeconomia e percorsi terapeutici 2005; 6 (2): 97-104

\section{INTRODUZIONE}

Il trattamento dei disturbi psicotici rappresenta un problema di complessa gestione per gli operatori sanitari.

I limiti dei vecchi neurolettici tipici e la cronica disabilità sociale caratterizzante queste patologie hanno promosso negli ultimi anni la diffusione di una nuova ed eterogenea classe di farmaci antipsicotici definiti "atipici” sia per i minori effetti avversi extrapiramidali sia per la maggiore efficacia verso i sintomi positivi e negativi della schizofrenia in particolare.

Sono state recentemente pubblicate alcune valutazioni farmacoeconomiche aventi come oggetto di studio il confronto fra atipici e neurolettici convenzionali. I risultati di questi studi hanno dimostrato che il maggior costo di
* Centro di

Psicofarmacoterapia -

Unità Operativa di Psichiatria "Lucio Bini" Azienda Ospedaliera

Policlinico-Consorziale

- Bari

$\S$ Health Outcomes Research - Eli Lilly Italia 
acquisto legato all'utilizzo di un nuovo atipico è più che bilanciato dal minore utilizzo di risorse sanitarie, in particolare i ricoveri ospedalieri [7], a seguito di una bassa incidenza di EPS e di una migliore compliance del paziente.

Con il presente lavoro si è voluto verificare tali fenomeni nell' ambito di una realtà psichiatrica locale, confrontando costi ed efficacia associati al trattamento di pazienti psicotici (schizofrenici e bipolari) con farmaci di vecchia e nuova generazione presso alcuni Servizi Psichiatrici della Regione Puglia. In particolare si è voluto valutare se l'utilizzo dei nuovi atipici rispetto alle terapie tradizionali comporti un impiego più efficiente delle risorse sanitarie.

\section{OBIETTIVI E PROSPETTIVA DI ANALISI}

Questo studio si propone di valutare e confrontare l'efficacia con i costi annuali di trattamento dei pazienti affetti da disturbi psicotici in regime di terapia con farmaci tipici ed atipici e, secondariamente, di confrontare tra loro, secondo lo stesso approccio, i quattro farmaci atipici a maggiore diffusione (clozapina, olanzapina, risperidone e quetiapina). Il miglioramento delle condizioni cliniche e generali dei pazienti (relativo anche alle relazioni sociali e al reinserimento nell'attività lavorativa) nel corso dei 12 mesi non è dovuto, ovviamente, solo al farmaco somministrato, ma anche agli interventi psicoterapeutici e riabilitativi. Tuttavia, il confronto avviene sotto l'ipotesi che, a parità di efficacia degli altri trattamenti, le differenze nei risultati siano attribuibili al tipo di farmaco impiegato.

Il punto di vista adottato per l'analisi farmacoeconomica è quello della ASL ed in particolare delle strutture erogatrici delle prestazioni psichiatriche. Alla luce di ciò, il consumo delle risorse sanitarie associato ai trattamenti antipsicotici è stato valorizzato utilizzando i costi reali di erogazione e non attraverso i costi indicati dai tariffari nomenclatori regionali.

\section{MATERIALI E METODI}

\section{Disegno dello studio e pazienti arruolati}

I dati su cui si è svolta la presente analisi farmacoeconomica provengono da uno studio osservazionale multicentrico della durata di 12 mesi condotto su 131 pazienti psicotici cronici (schizofrenici e bipolari) in trattamento con farmaci tipici ed atipici e seguiti da Strutture Psichiatriche pugliesi.

Sono stati arruolati pazienti affetti da disturbi psicotici (diagnosticati secondo il DSMIV) e trattati con neurolettici tipici (32 pazienti), olanzapina (46 pazienti), risperidone ( 25 pazienti), quetiapina (19 pazienti), clozapina (9 pazienti). I pazienti inclusi nel campione sono stati osservati per 12 mesi in un periodo compreso tra gli anni 2003 e 2004. L'osservazione si è svolta in senso prospettico per gran parte dei pazienti, mentre una minoranza è stata valutata retrospettivamente. Per questi ultimi i dati di efficacia e di assorbimento di risorse sanitarie sono stati estratti direttamente dalle relative cartelle cliniche archiviate presso le Strutture Psichiatriche di riferimento.

L'assegnazione dei pazienti ai cinque gruppi (neurolettici tipici, olanzapina, clozapina, risperidone, quetiapina) è avvenuta sulla base della terapia antipsicotica principale che, come requisito di arruolamento, doveva essere prescritta da almeno due mesi prima del periodo di osservazione.

I pazienti inclusi nell' analisi sono stati selezionati tra tutti i pazienti psicotici seguiti dai Servizi Psichiatrici pugliesi e inseriti nei vari gruppi di trattamento in modo che ciascun gruppo fosse confrontabile per età (uguale o superiore a 18 anni), gravità della patologia, omogeneità del nucleo familiare o di convivenza. Soltanto il gruppo di trattamento con clozapina ha incluso pazienti con gravità media più elevata e resistenti a precedenti trattamenti antipsicotici.

Al fine di poter stabilire un confronto tra $\mathrm{i}$ gruppi anche sulla base dell'outcome "attività lavorativa" si è posto attenzione a che le cinque coorti fossero confrontabili con riferimento alla condizione lavorativa dei pazienti (percentuali sovrapponibili nei gruppi di lavoratori, disabili, pensionati, disoccupati, etc.). Inoltre, sempre a tale scopo, si è considerata attività lavorativa anche quella svolta occasionalmente da pazienti affetti da disabilità croniche o da pensionati.

Sempre per rendere il più possibile confrontabili fra loro i cinque bracci di trattamento, nel gruppo neurolettici tipici non sono stati inclusi pazienti in terapia con formulazioni long-acting. Poiché infatti gli atipici non dispongono di preparazioni depot, ma solo ad assunzione quotidiana, si è ritenuto importante confrontarli con analoghe formulazioni di neurolettici tipici per non influenzare il peso della variabile compliance.

\section{Tipologia di dati raccolti}

In coerenza con l'obiettivo principale del lavoro sono stati raccolti dati sull'efficacia dei trattamenti e sui consumi di risorse sanitarie assorbite dai pazienti nel corso dei 12 mesi di osservazione.

I dati di efficacia sono stati raccolti valutando il re-inserimento nel "tessuto sociale", misurando in particolare la capacità del paziente di riprendere una regolare attività lavorativa, sintomo di miglioramento clinico associato al trattamento. 
La valutazione economica ha contemplato invece i costi diretti, ossia il consumo da parte del paziente psicotico di tutti i servizi e prestazioni sanitarie attribuibili direttamente alla malattia o al trattamento.

I dati raccolti sulle risorse sanitarie hanno compreso i consumi farmacologici relativi al trattamento della sola malattia psichiatrica (antipsicotico principale e associati, antidepressivi, anticolinergici, stabilizzatori dell'umore), i ricoveri e le prestazioni di assistenza territoriale quali:

a. giornate di riabilitazione in centro diurno,

b. visite specialistiche,

c. interventi infermieristici domiciliari e in struttura,

d. interventi medici domiciliari,

e. interventi di psicoterapia.

In linea con il punto di vista adottato per l'analisi, l'attribuzione dei costi è stata effettuata riferendosi a quelli reali di erogazione delle singole prestazioni, tratti da alcune recenti pubblicazioni in ambito psichiatrico ed attualizzati all'anno 2005 [2,5,6]. In particolare l'attribuzione dei costi ospedalieri è stata effettuata basandosi sui dati di costo per giornata di ricovero in SPDC.

I costi delle terapie farmacologiche sono stati invece individuati attraverso l'Informatore Farmaceutico Italiano, anno 2004 [8].

Una trattazione a parte è stata riservata ai costi relativi all'assistenza privata e alle giornate lavorative perse dal caregiver. Tali costi infatti, non rientrando tra quelli sostenuti dalle strutture erogatrici per il trattamento del paziente psicotico, non sono stati inclusi nell' analisi farmacoeconomica. Si è voluto tuttavia analizzarli separatamente in considerazione del loro elevato impatto sui familiari del paziente psicotico e sulla Società in generale.

Tutti i dati così raccolti sono stati trasferiti su foglio elettronico e quindi analizzati statisticamente per produrre valori medi di consumo e per valutare eventuali correlazioni tra tipo di trattamento ed outcome.

\section{RISULTATI}

\section{Efficacia}

In questa analisi farmacoeconomica si è proceduto a valutare l'efficacia attraverso la capacità dei diversi tipi di trattamento di riavviare il paziente psicotico all'attività lavorativa e, conseguentemente, di determinare un generale miglioramento delle condizioni cliniche.

L'avviamento all' attività lavorativa rappresenta del resto un parametro di efficacia sempre più importante ed utilizzato nelle valutazioni economiche sulle patologie psichiatriche, in quanto è espressione del livello di re-inseri-

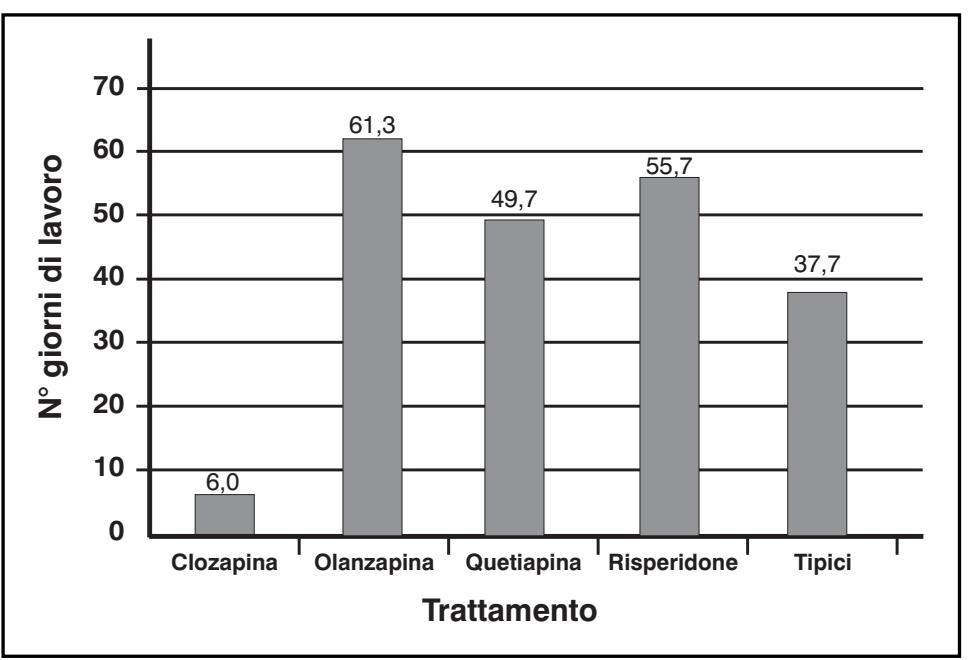

Figura 1

$N^{\circ}$ medio annuo di giornate lavorative a paziente per tipo di terapia (differenze non significative fra gruppi $p>0,05$ )

mento sociale del paziente e, conseguentemente, della diminuzione del carico economico sulle strutture sanitarie pubbliche.

L'attività lavorativa è stata misurata attraverso il numero medio annuo di giorni di lavoro per paziente associato a ciascun trattamento. I risultati non evidenziano differenze statisticamente significative fra i gruppi, dimostrando una efficacia sovrapponibile tra i trattamenti a confronto anche se, in termini di trend, olanzapina sembra denotare un vantaggio rispetto alle altre molecole (in media 61,3 giorni di lavoro a paziente in un anno). (Figura 1).

\section{Costi}

Dall'analisi dei costi medi annui per paziente per terapia farmacologica emerge un maggior costo per neurolettici (principali ed associati) nel gruppo quetiapina (5.094 euro), mentre il valore più basso si riscontra nel gruppo neurolettici tipici (551 euro) a causa del minor costo di acquisizione di questi farmaci rispetto agli atipici. Differenze statisticamente significative sul costo per neurolettici vengono evidenziate su tutti i confronti diretti fra gruppi (Tabella I).

I valori medi di costo più elevati per farmaci neurolettici si sono riscontrati nei gruppi quetiapina (5.094 euro) e olanzapina (2.839 euro) (Tabella I). Ciò è attribuibile al maggior costo di acquisizione del farmaco per queste due molecole.

Per quanto concerne i costi relativi alle terapie associate non si riscontrano differenze statisticamente significative tra i gruppi sull'utilizzo di antidepressivi e stabilizzatori dell'umore, mentre valori irrilevanti di costo presentano le associazioni con anticolinergici (Tabella I).

Il computo totale del costo degli interventi farmacologici vede il maggior onere economico nel gruppo quetiapina (5.283 euro), seguito dai gruppi olanzapina (3.066 euro), clozapina (2.148 euro), risperidone (1.906 euro) e neurolettici ti- 


\section{Tabella I}

Costi medi annui a paziente per farmaci per tipo di trattamento (dati in euro)

\begin{tabular}{lccccc}
\hline & $\begin{array}{c}\text { Totale } \\
\text { Neurolettici } \\
\text { Principale }+ \text { associato }\end{array}$ & $\begin{array}{c}\text { Totale } \\
\text { Antidepressivi }\end{array}$ & $\begin{array}{c}\text { Totale } \\
\text { Anticolinergici }\end{array}$ & $\begin{array}{c}\text { Totale } \\
\text { Stabilizzatori } \\
\text { Umore }\end{array}$ & $\begin{array}{c}\text { Totale } \\
\text { Farmaci }\end{array}$ \\
\hline Clozapina & 1.854 & 230 & 0 & 65 & 2.148 \\
Olanzapina & 2.839 & 144 & 10 & 72 & 3.066 \\
Quetiapina & 5.094 & 118 & 11 & 62 & 5.283 \\
Risperidone & 1.722 & 61 & 13 & 110 & 1.906 \\
Tipici & 551 & 107 & 34 & 41 & 734 \\
Significatività & & & & & \\
C-O & 0,0177 & $n$ & $n$ & $n s$ & 0,0280 \\
O-Q & $<0,0001$ & $n s$ & $n s$ & $n s$ & $<0,0001$ \\
O-R & 0,0001 & $n s$ & $n s$ & $n s$ & $<0,0001$ \\
O-T & $<0,0001$ & $n s$ & $<0,0001$ & $n s$ & $<0,0001$ \\
Q-R & $<0,0001$ & $n s$ & $n s$ & $n s$ & $<0,0001$ \\
Q-T & $<0,0001$ & $n s$ & 0,0014 & $n s$ & $<0,0001$ \\
R-T & 0,0002 & $n s$ & 0,0017 & $n s$ & 0,0002 \\
\hline
\end{tabular}

pici (734 euro). Differenze statisticamente significative si rilevano su tutti i confronti diretti fra i gruppi (Tabella I). I costi totali farmacologici non includono il costo di acquisizione delle benzodiazepine perché distribuite in classe $\mathrm{C}$ e quindi a carico del paziente.

Se dai farmaci spostiamo l'attenzione ai costi relativi alle risorse sanitarie, $i$ dati evidenziano come il valore più basso venga associato ad olanzapina (5.778 euro) mentre il picco più alto si riscontra su clozapina (12.293 euro). Tuttavia i confronti diretti fra gruppi su questa voce di costo non generano differenze statisticamente significative, ad eccezione del confronto olanzapina-clozapina ( $\mathrm{p}=0,0041)$. Questo risultato sta ad indicare che l'assorbimento di risorse sanitarie non farmacologiche per la gestione

\begin{tabular}{lccc} 
& Farmaci & Risorse sanitarie & Totale \\
\hline Clozapina & 2.148 & 12.293 & 14.441 \\
Olanzapina & 3.066 & 5.778 & 8.843 \\
Quetiapina & 5.283 & 7.061 & 12.344 \\
Risperidone & 1.906 & 6.998 & 8.904 \\
Tipici & 734 & 7.304 & 8.038 \\
& & & \\
Significatività & & & \\
C-O & 0,0280 & 0,0041 & 0,0156 \\
O-Q & $<0,0001$ & $\mathrm{~ns}$ & 0,0426 \\
O-R & $<0,0001$ & $\mathrm{~ns}$ & $\mathrm{~ns}$ \\
O-T & $<0,0001$ & $\mathrm{~ns}$ & $\mathrm{~ns}$ \\
Q-R & $<0,0001$ & $\mathrm{~ns}$ & $\mathrm{~ns}$ \\
Q-T & $<0,0001$ & $\mathrm{~ns}$ & 0,0192 \\
R-T & 0,0002 & $\mathrm{~ns}$ & $\mathrm{~ns}$ \\
\hline
\end{tabular}

\section{Tabella II}

Costi totali medi di trattamento del paziente psicotico cronico per tipo di terapia del paziente psicotico genera dei costi che, mediamente, sono sovrapponibili fra i diversi trattamenti, ad eccezione del confronto clozapinaolanzapina che denota un vantaggio economico per quest'ultima (Tabella II).

Ci preme inoltre sottolineare come i maggiori costi di prestazioni sanitarie associati a clozapina siano influenzati dalla gravità media più elevata presente in questo gruppo rispetto agli altri trattamenti. Infatti la percentuale di pazienti classificati secondo la CGI nelle fasce ad elevata gravità ("gravi" e "molto gravi") all'arruolamento era così distribuita nei gruppi: clozapina 55,6\%, olanzapina $37,0 \%$, quetiapina $31,6 \%$, risperidone $40,0 \%$, neurolettici tipici $40,6 \%$.

La sommatoria delle singole voci di costo (farmaci + risorse sanitarie) nei cinque gruppi determina il costo globale di trattamento del paziente psicotico nonché il relativo carico economico complessivo per le Strutture Sanitarie pubbliche. Tali costi evidenziano un vantaggio, statisticamente significativo, di olanzapina (8.843 euro) rispetto sia a quetiapina (12.344) $(\mathrm{p}=0,0426)$, che a clozapina $(14.441)(\mathrm{p}=0,0156)$. Significatività statistica $(\mathrm{p}=0,0192)$ caratterizza anche il confronto neurolettici tipici (8.038) vs quetiapina (12.344). Gli altri confronti non denotano invece differenze statisticamente significative (Tabella II).

I dati evidenziati in termini di costi globali, unitamente ai risultati sovrapponibili in termini di efficacia (espressa dall'attitudine lavorativa del paziente) delineano una sostanziale equivalenza tra i trattamenti antipsicotici confrontati nella realtà pugliese. Nei confronti diretti fra i gruppi l'applicazione del modello di minimizzazione dei costi delinea soltanto un vantaggio di olanzapina rispetto a quetiapina e clozapina in quanto, a parità di efficacia, essa presenta costi totali di trattamento significati- 
vamente inferiori $(\mathrm{p}<0,05)$. Lo stesso dicasi per il confronto tipici vs quetiapina con un vantaggio di costo statisticamente significativo $(\mathrm{p}=0,0192)$ a favore dei vecchi neurolettici.

Vogliamo tuttavia focalizzare l' attenzione sui costi non farmacologici, ovvero relativi a tutte le risorse sanitarie extra-farmaco necessarie alla cura del paziente psicotico. Essi rappresentano, alla luce dei risultati dello studio multicentrico pugliese, l' $80 \%$ del costo complessivo di trattamento del paziente psicotico cronico (Figura 2).

Ne consegue l'importanza di adottare strategie terapeutiche in grado di ottimizzare l'efficienza di utilizzo di tali risorse abbattendone contemporaneamente i costi. La Tabella III mostra il consumo medio a paziente di risorse sanitarie per tipo di trattamento nell' ambito del primo (T180) e secondo (T360) semestre di osservazione con relative differenze e test di significatività. In quest'ottica olanzapina risulta essere l'unica scelta terapeutica in grado di ridurre, in maniera statisticamente significativa, fra primo e secondo semestre, il consumo medio di tutte le principali risorse sanitarie utilizzate per il trattamento del paziente psicotico (Tabella III).

Questo risultato emerso dalla ricerca è indicativo, oltre che di un impiego più efficiente delle risorse sanitarie collegato al trattamento con olanzapina, anche di una migliore "performance clinica" e risocializzante, a lungo termine, sul paziente psicotico cronico.

Se si considera la totalità delle risorse sanitarie che mediamente si rendono necessarie per trattare il paziente psicotico cronico, non vi è dubbio che i ricoveri rappresentino la voce di costo a maggiore impatto per i bilanci delle Aziende Sanitarie pubbliche della Regione Puglia (Figura 3).

La Figura 4 sintetizza la diversa propensione dei trattamenti a confronto nel generare

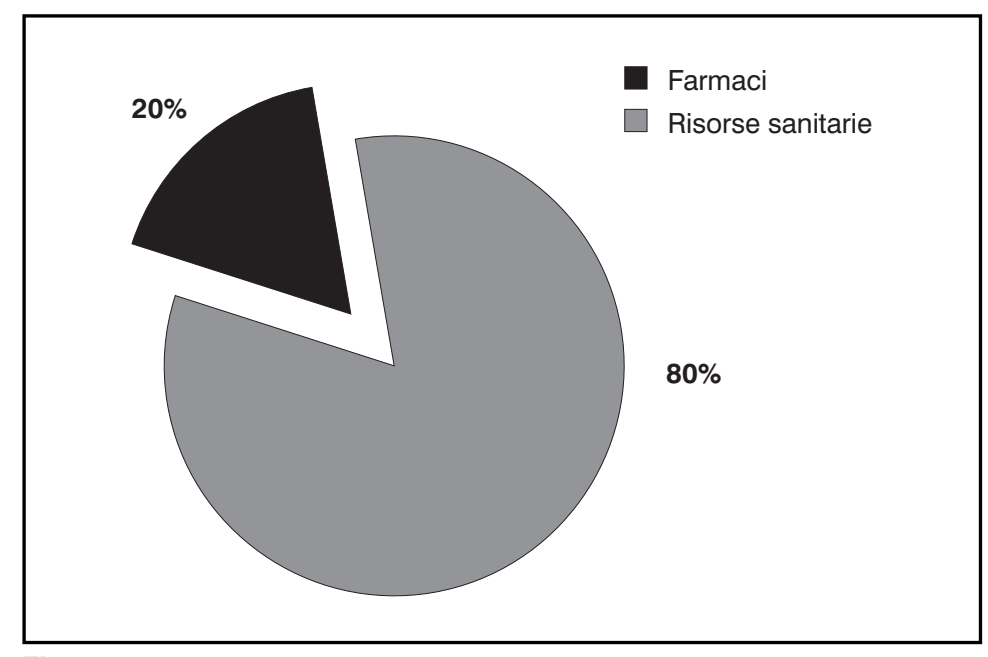

Figura 2

Mix costo totale medio di trattamento del paziente psicotico

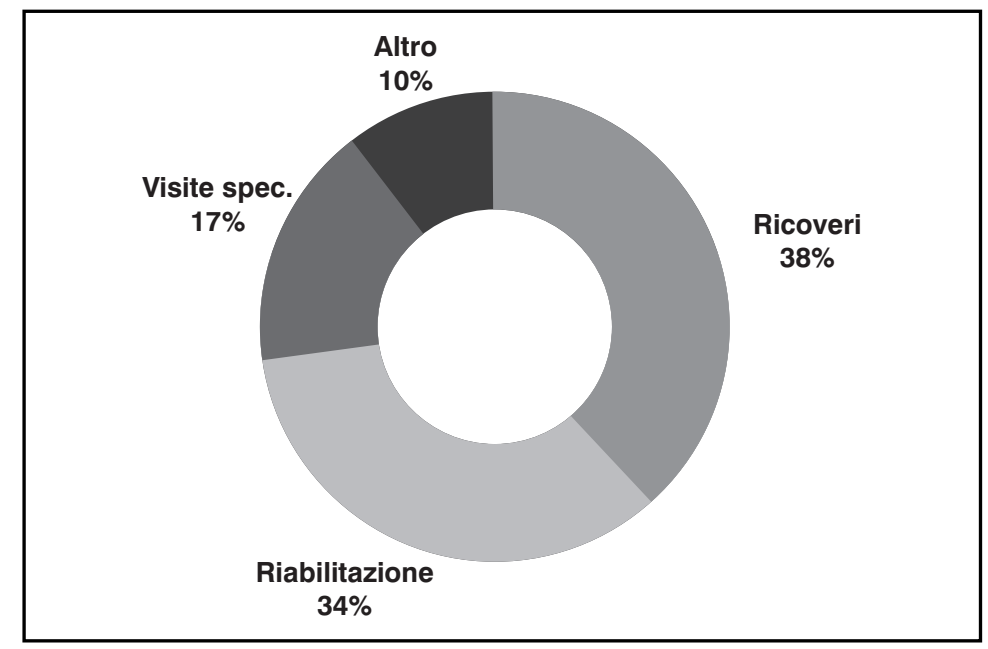

Figura 3

Mix consumo medio di risorse sanitarie per il trattamento del paziente psicotico

ospedalizzazioni del paziente psicotico. L'asse delle ascisse misura il numero medio di giorna-

\begin{tabular}{|c|c|c|c|c|c|c|}
\hline & & $\begin{array}{c}\text { Visite } \\
\text { specialistiche }\end{array}$ & $\begin{array}{l}\text { Interventi } \\
\text { inferm. } \\
\text { domiciliari }\end{array}$ & $\begin{array}{c}\text { Interventi } \\
\text { medici } \\
\text { domiciliari }\end{array}$ & $\begin{array}{l}\text { Interventi di } \\
\text { psicoterapia }\end{array}$ & $\begin{array}{l}\text { Giornate di } \\
\text { ricovero }\end{array}$ \\
\hline Olanzapina & $\begin{array}{l}\text { T180 } \\
\text { T360 } \\
\text { Differenza } \\
p\end{array}$ & $\begin{array}{c}11,78 \\
9,30 \\
-2,48 \\
\mathbf{0 , 0 1 2 2}\end{array}$ & $\begin{array}{c}1,02 \\
0,41 \\
-0,61 \\
\mathbf{0 , 0 3 8 6}\end{array}$ & $\begin{array}{c}1,15 \\
0,46 \\
-0,70 \\
\mathbf{0 , 0 0 5 9}\end{array}$ & $\begin{array}{c}2,52 \\
1,59 \\
-0,93 \\
\mathbf{0 , 0 3 0 1}\end{array}$ & $\begin{array}{c}4,63 \\
0,59 \\
-4,04 \\
\mathbf{0 , 0 1 7 3}\end{array}$ \\
\hline Quetiapina & $\begin{array}{l}\text { T180 } \\
\text { T360 } \\
\text { Differenza } \\
\text { p }\end{array}$ & $\begin{array}{c}11,95 \\
10,26 \\
-1,68 \\
0,0860\end{array}$ & $\begin{array}{c}0,74 \\
0,74 \\
0,00 \\
1,0000\end{array}$ & $\begin{array}{c}0,84 \\
0,58 \\
-0,26 \\
0,4710\end{array}$ & $\begin{array}{c}4,53 \\
3,37 \\
-1,16 \\
0,2610\end{array}$ & $\begin{array}{c}6,63 \\
0,89 \\
-5,74 \\
0,2509\end{array}$ \\
\hline Risperidone & $\begin{array}{l}\text { T180 } \\
\text { T360 } \\
\text { Differenza } \\
p\end{array}$ & $\begin{array}{c}11,32 \\
10,08 \\
-1,24 \\
0,1355\end{array}$ & $\begin{array}{c}0,84 \\
0,92 \\
0,08 \\
0,6469\end{array}$ & $\begin{array}{c}1,04 \\
0,44 \\
-0,60 \\
0,1546\end{array}$ & $\begin{array}{c}3,44 \\
2,24 \\
-1,20 \\
0,0580\end{array}$ & $\begin{array}{c}5,56 \\
0,00 \\
-5,56 \\
\mathbf{0 , 0 1 4 4}\end{array}$ \\
\hline Clozapina & $\begin{array}{l}\text { T180 } \\
\text { T360 } \\
\text { Differenza } \\
p\end{array}$ & $\begin{array}{c}8,33 \\
8,67 \\
0,33 \\
0,8307\end{array}$ & $\begin{array}{c}0,56 \\
0,78 \\
0,22 \\
0,8315\end{array}$ & $\begin{array}{c}0,56 \\
0,67 \\
0,11 \\
0,7995\end{array}$ & $\begin{array}{c}0,22 \\
0,00 \\
-0,22 \\
0,3466\end{array}$ & $\begin{array}{c}19,89 \\
0,00 \\
-19,89 \\
0,2141\end{array}$ \\
\hline N. Tipici & $\begin{array}{l}\text { T180 } \\
\text { T360 } \\
\text { Differenza } \\
\text { p }\end{array}$ & $\begin{array}{c}11,59 \\
11,88 \\
0,28 \\
0,5827\end{array}$ & $\begin{array}{c}2,06 \\
1,81 \\
-0,25 \\
0,5679\end{array}$ & $\begin{array}{c}2,00 \\
1,22 \\
-0,78 \\
0,0559\end{array}$ & $\begin{array}{c}0,97 \\
1,19 \\
0,22 \\
0,2562\end{array}$ & $\begin{array}{c}6,28 \\
4,97 \\
-1,31 \\
0,5549\end{array}$ \\
\hline
\end{tabular}

Tabella III

Consumo medio annuo a paziente di risorse sanitarie per tipo di trattamento: differenze fra l e II semestre di osservazione 
te di ricovero all'anno per paziente associate a ciascuna terapia; le ordinate riportano invece il grado di significatività statistica con cui ciascuna scelta di trattamento riduce le giornate di ricovero fra primo e secondo semestre di osservazione; infine le dimensioni delle bolle indicano il costo medio annuo sostenuto dalla struttura per le giornate di ricovero a paziente associate a ciascun trattamento. I dati non evidenziano differenze statisticamente significative fra i trattamenti sul numero medio di giorni di ricovero né sui relativi costi medi annuali; uniche eccezioni i confronti olanzapina-clozapina $(\mathrm{p}=0,0309)$ e risperidone-clozapina $(\mathrm{p}=0,0477)$ ove la clozapina risulta sempre in svantaggio anche se, come detto in precedenza, è stata utilizzata su pazienti mediamente più gravi.

Nonostante tale risultato, dalla Figura 4 si evince che il numero di giornate di ricovero cala, in maniera statisticamente significativa fra primo e secondo semestre, solo con i trattamenti olanzapina $(\mathrm{p}=0,0173)$ e risperidone $(\mathrm{p}=0,0144)$. Se si considera inoltre che, come evidenziato in Tabella III, questo fenomeno si riscontra, solo per olanzapina, anche su tutti gli altri tipi di risorse impiegate (visite specialistiche, interventi medici domiciliari, etc.) è lecito considerare olanzapina l'opzione terapeutica che garantisce la migliore efficienza di trattamento sul paziente psicotico cronico.

Ci preme infine soffermare l'attenzione sui costi indiretti (giornate lavorative perse dal caregiver) e su quelli legati all'assistenza privata. In linea con la prospettiva di analisi del presente lavoro, essi non sono stati inclusi nella valutazione farmacoeconomica e non rientrano nel computo dei costi totali di trattamento del paziente psicotico, in quanto non sostenuti direttamente dalle Strutture Sanitarie pub-

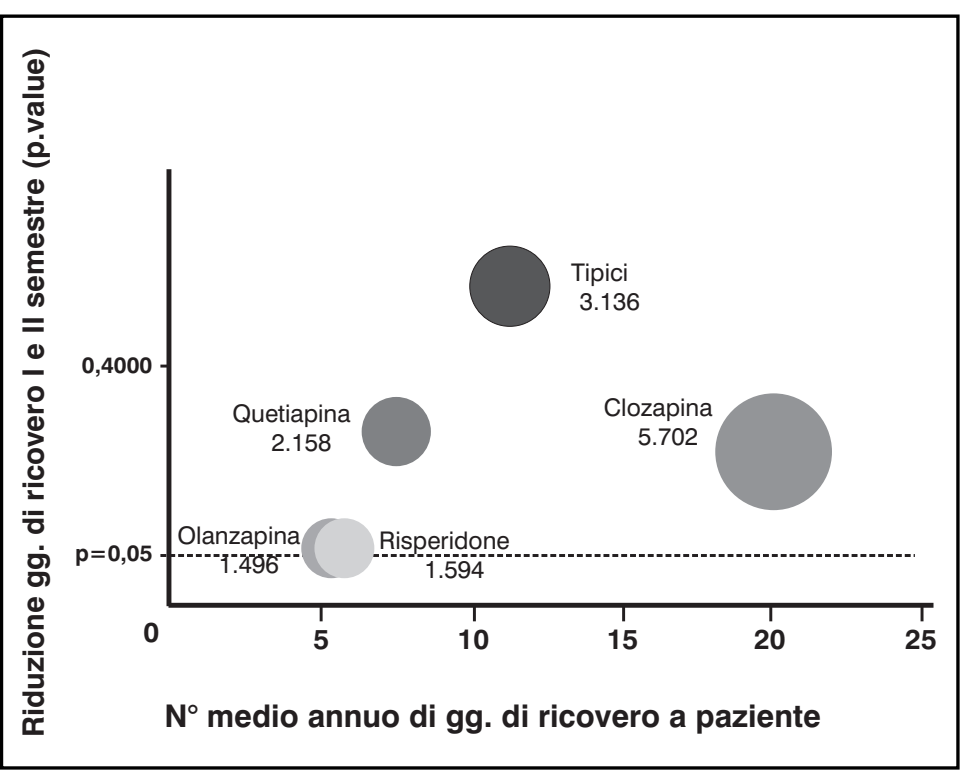

Figura 4

Giorni di ricovero a paziente per tipo di trattamento e relativi costi bliche. Tali costi meritano comunque una considerazione particolare alla luce del loro rilevante impatto economico sui familiari del paziente e sulla Società in generale.

A tal riguardo i dati raccolti nella realtà psichiatrica pugliese confermano la superiorità di olanzapina in termini di efficienza di trattamento. Essa rappresenta infatti l'unica scelta terapeutica in grado di ridurre, in maniera statisticamente significativa $(\mathrm{p}=0,0315)$, le giornate lavorative perse dal caregiver fra primo e secondo semestre di osservazione (Tabella IV) evitando contemporaneamente al paziente e ai suoi familiari il ricorso all'assistenza privata (Figura 5). Gli altri trattamenti a confronto evidenziano invece o riduzioni non significative dell'utilizzo del caregiver (quetiapina e clozapina - Tabella IV) oppure l'incapacità di evitare costi legati all'assistenza privata del paziente psicotico (risperidone e tipici - Figura 5).

\section{DISCUSSIONE}

La terapia farmacologica con neurolettici è senza alcun dubbio riconosciuta come il "gold standard" per il trattamento dei disturbi psicotici e della schizofrenia in particolare. Tuttavia l'insorgenza di gravi eventi avversi legati all'utilizzo dei neurolettici convenzionali ha indotto la ricerca farmacologica a sperimentare gli antipsicotici atipici che, a parità di efficacia, presentano un profilo di tollerabilità estremamente migliore rispetto ai neurolettici tradizionali e per questo sono sempre più utilizzati nella pratica clinica. Nonostante l'interesse crescente per questa nuova categoria terapeutica, sono ancora pochi gli studi di comparazione diretta tra questi. L'analisi della letteratura ha infatti evidenziato che la maggior parte delle comparazioni avviene, sia in termini clinici che economici, con i neurolettici tradizionali (ad es. aloperidolo) [12].

Il presente lavoro intende contribuire alle conoscenze sui costi e l'efficacia di trattamento dei disturbi psicotici in Italia focalizzando l'analisi sul confronto diretto fra tutti gli atipici disponibili attualmente nella pratica clinica e tra questi e i neurolettici tipici.

Lo studio, di tipo osservazionale, ha esaminato lungo un periodo di 12 mesi i costi globali e l'efficacia di trattamento su 131 pazienti psicotici cronici (schizofrenici e bipolari) in terapia con farmaci tipici e atipici e in carico presso Servizi Psichiatrici della Regione Puglia. Nei 12 mesi di osservazione sono stati valutati sia il periodo di mantenimento, durante il quale i pazienti afferiscono a Strutture Psichiatriche territoriali, sia i periodi di ricovero in ospedale che intervengono quando i pazienti presentano fasi di riacutizzazione della patologia.

Le variabili di assorbimento delle risorse sanitarie che sono state rilevate hanno com- 
preso tutti gli interventi farmacologici intrapresi nell'anno di osservazione come terapia principale e associata, interventi delle strutture territoriali (visite specialistiche, visite mediche domiciliari, interventi infermieristici in struttura e domiciliari, colloqui con lo psicologo, riabilitazione) e i ricoveri.

L'efficacia dei trattamenti è stata invece valutata attraverso il reinserimento del paziente psicotico nell'attività lavorativa quale espressione del miglioramento generale delle condizioni cliniche.

La scelta di procedere attraverso uno studio osservazionale è stata dettata dall'esigenza di voler produrre dei risultati che rispecchiassero fedelmente la pratica clinica seguita all'interno delle Strutture Psichiatriche.

Il pool di pazienti è stato selezionato e incluso nei cinque bracci di trattamento (olanzapina, risperidone, quetiapina, clozapina, neurolettici tipici) in base alla terapia antipsicotica principale seguita. Inoltre i pazienti sono stati arruolati nei gruppi in modo che questi fossero sovrapponibili per età media, gravità della patologia e condizioni socio-lavorative.

Nonostante ciò, è risultato che il gruppo clozapina presentasse una gravità media all'arruolamento superiore agli altri gruppi e includesse pazienti resistenti a precedenti trattamenti antipsicotici. Infatti la percentuale di pazienti classificati secondo la CGI nelle fasce ad elevata gravità ("gravi" e "molto gravi") all'arruolamento era così distribuita nei gruppi: clozapina $55,6 \%$; olanzapina $37,0 \%$; quetiapina $31,6 \%$; risperidone $40,0 \%$; neurolettici tipici $40,6 \%$. Il punteggio medio CGI relativo alla gravità della patologia presentava invece sempre all'arruolamento i seguenti valori per gruppo di terapia: clozapina 5,22 ; olanzapina 4,76 ; quetiapina 4,47 ; risperidone 5,08; neurolettici tipici 4,91.

Un altro possibile bias nel confronto fra terapie antipsicotiche può essere stato indotto dalla numerosità non sovrapponibile di pazienti all'interno delle cinque coorti. I gruppi infatti risultavano così popolati per numero di pazienti: olanzapina 46, neurolettici tipici 32, risperidone 25 , quetiapina 19 , clozapina 9 .

I risultati dello studio svoltosi con i criteri sopra indicati delineano una sostanziale parità di efficacia (valutata attraverso il reinserimento socio-lavorativo del paziente) fra i gruppi, in quanto il computo del numero medio annuo a paziente di giornate dedicate al lavoro non conduce a differenze statisticamente significative (Figura 1).

Sul piano economico, coerentemente con quanto già pubblicato in esperienze internazionali e non $[1,3,11]$, il maggior costo di acquisizione del farmaco principale per gli atipici è in genere compensato dal minor ricorso al consumo di risorse sanitarie (ricoveri ed interventi

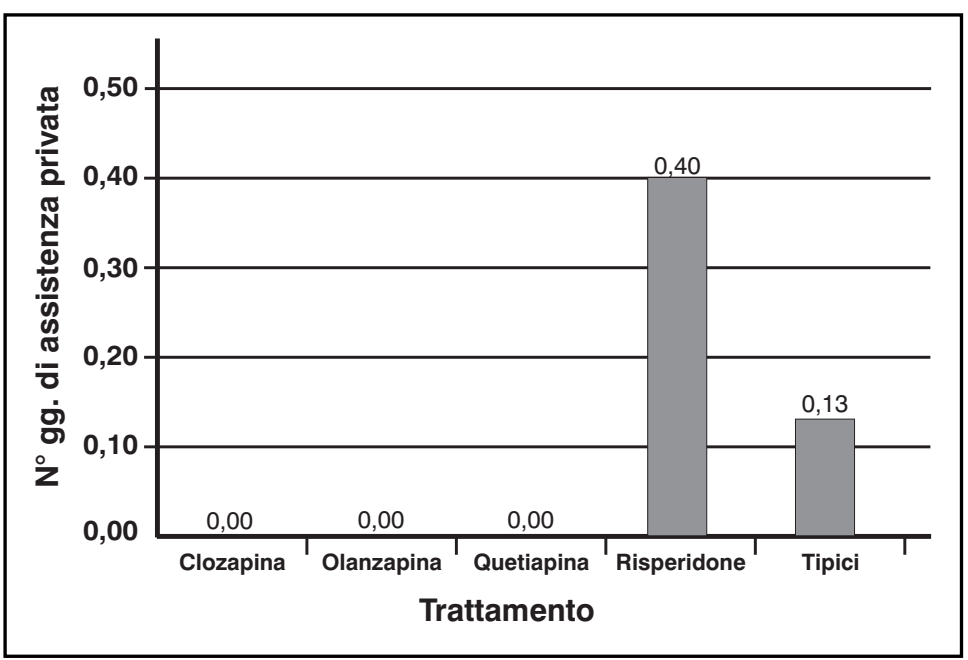

Figura 5

Numero medio annuo a paziente di giornate di assistenza privata per tipo di terapia

\begin{tabular}{lcccc}
\hline & T180 & T360 & Differenza & p value \\
\hline Clozapina & 5,22 & 0,56 & $-4,67$ & 0,195 \\
Olanzapina & 4,07 & 2,33 & $-1,74$ & $\mathbf{0 , 0 3 1 5}$ \\
Quetiapina & 8 & 6,42 & $-1,58$ & 0,1384 \\
Risperidone & 2,88 & 1 & $-1,88$ & $\mathbf{0 , 0 3 2 7}$ \\
Tipici & 4,94 & 2,31 & $-2,63$ & $\mathbf{0 , 0 1 9 4}$ \\
\hline
\end{tabular}

\section{Tabella IV}

Numero medio a paziente di giornate lavorative perse dal caregiver: differenze fra l e I/ semestre di osservazione

territoriali) rispetto ai tipici. Questo fenomeno fa sì che, in un'ottica di costi totali di trattamento, nella maggior parte dei confronti diretti fra gruppi, non si rilevino differenze statisticamente significative (Tabella II).

Olanzapina risulta comunque essere l'alternativa più vantaggiosa nel confronto diretto con quetiapina in quanto, a parità di efficacia, i costi totali di trattamento (in media 8.843 euro all' anno per paziente) risultano significativamente inferiori $(\mathrm{p}=0,0426)$ rispetto al gruppo quetiapina (in media 12.344 euro all'anno per paziente). Quetiapina risulta essere l'opzione meno vantaggiosa $(p=0,0192)$ anche nel confronto diretto con i neurolettici tipici (per i quali si rileva un costo totale medio annuo a paziente pari a 8.038 euro) (Tabella II).

Scarsamente indicativi risultano invece $\mathrm{i}$ confronti diretti con il gruppo clozapina in considerazione della gravità media più elevata di questi pazienti, che induce ad un consumo maggiore di risorse sanitarie.

I risultati della presente valutazione farmacoeconomica hanno evidenziato che il consumo di risorse sanitarie non farmacologiche (ricoveri, riabilitazione, visite specialistiche, interventi medici domiciliari, etc.) rappresenta l' $80 \%$ dei costi totali di trattamento del paziente psicotico cronico (Figura II). 
Ne consegue l'importanza strategica per le Strutture Sanitarie pubbliche di ottimizzare il consumo di tali risorse adottando scelte terapeutiche che ne riducano l'utilizzo sul lungo termine. In questo senso lo studio ha dimostrato che olanzapina rappresenta l'unica terapia antipsicotica in grado di ridurre, sul lungo termine e in modo statisticamente significativo, il consumo di tutte le risorse sanitarie non farmacologiche necessarie al trattamento dei disturbi psicotici (Tabella III). Tale fenomeno è sintomatico di un contributo determinante al miglioramento del quadro clinico dei pazienti, oltre che di una più efficiente gestione delle risorse sempre più scarse in ambito sanitario.

Questi risultati a favore di olanzapina vengono confermati anche dalla disamina sui costi indiretti e relativi all'assistenza privata al paziente psicotico cronico; nell'ambito degli antipsicotici confrontati, olanzapina risulta l'unico trattamento che associa ad una riduzione statisticamente significativa sul lungo termine delle giornate lavorative perse dal caregiver (Tabella IV) una totale assenza di costi sostenuti dai familiari per l'assistenza privata al paziente (Figura 5).
In conclusione, la nostra analisi evidenzia che il trattamento dei pazienti psicotici con alcuni atipici (olanzapina e risperidone) non è economicamente più oneroso di quello con neurolettici tipici; l'utilizzo invece di altri atipici (quetiapina) comporta costi totali significativamente superiori $(\mathrm{p}=0,0192)$ a quelli dei vecchi neurolettici.

All'interno degli atipici, escludendo clozapina per i motivi sopra indicati, si riscontra invece un sostanziale equilibrio in termini sia di efficacia che di costi totali di trattamento; unica eccezione il confronto diretto quetiapina-olanzapina che vede un vantaggio statisticamente significativo $(\mathrm{p}=0,0426)$ in termini di costo totale a favore di quest'ultima.

$\mathrm{Al}$ di là di queste considerazioni sembra comunque di poter affermare che olanzapina risulti in assoluto l'antipsicotico più vantaggioso fra quelli confrontati nella realtà pugliese alla luce del consumo delle prestazioni sanitarie e dei costi di assistenza che, con il suo utilizzo, calano in maniera significativa sul lungo termine, ad espressione di un evidente miglioramento clinico del paziente, di un impiego più efficiente delle risorse e di un minore carico economico/assistenziale per i familiari e per la Società in generale.

\section{BIBLIOGRAFIA}

1. Almond S., O'Donnel O. Cost analysis of the treatment of schizophrenia in the UK, a comparison of olanzapine and haloperidol. Pharmacoeconomics, 1998, 13 (5 pt 2): 575-588

2. Arrighi E. et al. Pattern di trattamento e costi nei dipartimenti di salute mentale: il progetto di Ricerca HoNOS 2 , Il Pensiero Scientifico Editore, 2002: 58-60

3. Berardi D. et al. Costo del trattamento con olanzapina nei Servizi di Salute Mentale. Atti Congresso SIP 2000, pag. 820

4. Drummond MF, O’Brien BJ, Stoddart GL, et al. (Edizione italiana a cura di Fattore G, Garattini L, Lucioni C) Metodi per la valutazione economica dei programmi sanitari. Il Pensiero Scientifico Editore, 2000

5. Fattore G. et al. I costi delle prestazioni in psichiatria, Epidemiologia e Psichiatria Sociale, 1997, 6(2): 139-147

6. Garattini L. et al. ASI, 1996, n.10:33-37

7. Guest J.F., Hart W.M., Cookson R.F., et al. Pharmacoeconomic evaluation of long-term treatment with risperidone for patients with chronic schizofrenia, British Journal of Medical Economics, 1996; 10: 59-67

8. L'Informatore farmaceutico 2004. OEMF, 65a edizione

9. Mapelli V. et al. Costi ed efficacia della cura della schizofrenia con antipsicotici tipici ed atipici, PharmacoEconomics Italian Research Articles, 2003; vol. 5, n. 3, 1-15

10. Murray R.M., Jones P.B., Susser E., et al. The Epidemiology of Schizophrenia. Cambridge: Cambridge University Press, 2003

11. Rossi I., Guidi L. Valutazione dei costi di trattamento dei disturbi psicotici con olanzapina, risperidone e neurolettici tipici di un DSM italiano, Farmeconomia e percorsi terapeutici, 2001; 2 (4): 253-258

12. Tarricone R., Valutazione di convenienza economica comparata tra quetiapina e risperidone nel trattamento della schizofrenia, PharmacoEconomics Italian Research Articles, 2001; vol. 3, n. 1, 27-36

13. Tarricone R., Valutazioni economiche e management in sanità: applicazioni ai programmi e tecnologie sanitarie; Collana Management e Sanità; Mc Graw Hill, 2004 